\title{
Review of: "Experimental Investigation of Pulse Sterilization of Pathogens"
}

\author{
Koichi Takaki ${ }^{1}$ \\ 1 Iwate University \\ Potential competing interests: The author(s) declared that no potential competing interests exist.
}

The experimental result includes useful information for researchers in same research field. However, it is difficult to understand the threshold of UV dose for killing bacteria. The apparatus of MPC UV radiation system and input energy to produce UV emission are unclear. The additional information and description about the UV radiation apparatus and threshold of UV dose for killing bacteria are desirable for understanding the work clearly by the readers. 